vibrations, crystal defects, the presence of isotopes, and the different orientations of the nuclear spin. The experiment, therefore, fixes an upper limit to the residual scattering caused by the presence of several isotopes in lead:

$$
s_{\text {is }} \leqslant 0.6 \pm 0.3 \text { barn, }
$$

which does not disagree with the upper limits of 1.6 and 2.5 barns set by transmission measurements ${ }^{1,2}$.

If we use the scattering length $a$ as defined by Fermi ${ }^{3}$, the normal random scattering cross-section is given by

$$
\sigma=4 \pi \Sigma p_{r} a_{r}{ }^{2}
$$

and the coherent scattering cross-section relevant in single crystal diffraction is

$$
\mathbf{s}=4 \pi\left(\sum_{r} p_{r} a_{r}\right)^{2}
$$

The three cross-sections are related by the simple equation :

$$
s_{\text {is }}=\sigma-\mathbf{s}=4 \pi\left(\overline{a^{2}}-a^{2}\right) .
$$

Since $s_{\text {is }}$ is so small, it is evident that the minimum value of $\mathrm{s}$ is about 10 barns, and so the scattering lengths of all the major lead isotopes must be nearly equal. This is reasonable, since the small capture cross-section shows the absence of nearby resonances.

This result is in disagreement with the tentative value for $s$ of 2.9 barns deduced by Fermi and Marshall ${ }^{3}$ from measurements on a lead sulphide crystal. Substitution in their results of form factors modified in accordance with $s=10$ barns certainly gives an uneven decrease of intensity with order of reflexion, and it is not clear at present how this arises.

Cavendish Laboratory,

Cambridge. Nov. 14.

'Fermi, E., and Marshall, L., Phys. Rev., 72, 408 (1947).

'Havens, W. W., Rabi, I. I., and Rainwater, L. J., Phys. Rev., '2, 634 (1947).

${ }^{2}$ Fermi, E., and Marshall, L., Phys. Rev., 71, 666 (1947).

\section{Production of Nitrogen Atoms in the Upper Atmosphere}

EVIDENCE has accumulated in recent years that nitrogen is at least partially dissociated in the upper atmosphere. However, thus far the precise process causing the dissociation of nitrogen has not been established, except that it has been suggested that only the extremely short ultra-violet beyond $500 \mathrm{~A}$. could produce it ${ }^{1}$.

Recently, R. Herman ${ }^{2}$, under certain conditions of discharge, found a breaking off above the level $v^{\prime}=6$ in the Lyman-Birge-Hopfield $(a-X)$ bands of nitrogen. We have confirmed this breaking off in nitrogen excited at low pressure in a high-frequency electrodeless discharge. The appearance of our spectra leaves no doubt that we have here a predissociation of the $\mathrm{N}_{2}$ molecule. The predissociation limit is between the levels $v^{\prime}=6$ and 7 of the $a^{1} \Pi_{\mathrm{g}}$ state at an energy of approximately $9.8 \mathrm{eV}$., which agrees closely with the energy necessary to dissociate $\mathrm{N}_{2}$ into $\mathrm{N}\left({ }^{4} S\right)$ and $\mathrm{N}\left({ }^{2} D\right)$, assuming $D\left(\mathrm{~N}_{2}\right)=7 \cdot 38 \mathrm{eV}$. The $a-X$ bands are the only absorption bands of $\mathrm{N}_{2}$ above $1150 \mathrm{~A}$. (see Birge and Hopfield ${ }^{3}$ ). The absorption is comparatively weak, since the bands represent a forbidden transition (see G. Herzberg ${ }^{4}$ ).

The $v^{\prime \prime}=0$ progression has been observed in absorption from the $0-0$ to the $9-0$ band. The 7-0, $8-0$ and $9-0$ bands have the wave-lengtins $1249 \cdot 3$,
1226.6 and 1205.3 A. respectively. Absorption of solar radiation of these wave-lengths will lead to the production of nitrogen atoms in the upper atmosphere, since the molecules in the upper levels will undergo predissociation. The observation that in the laboratory the predissociation becomes noticeable only at low pressures indicates that the predissociation is a forbidden one. Nevertheless, in the upper atmosphere, a considerable fraction of the excited molecules will predissociate rather than return to the ground-state with emission of fluorescence.

On account of the comparatively low transition probability of the $a-X$ bands, the formation of nitrogen atoms according to the above mechanism will go on over a very considerable range of heights, particularly since in the region 1150-1250 A. absorption by oxygen molecules is comparatively weak. It is thus possible to account both for the presence of nitrogen atoms at comparatively low heights and for the presence of nitrogen molecules at much greater heights (as observed in auroras).

While it is conceivable that nitrogen atoms may also be produced at much shorter wave-lengths through different mechanisms, we feel, in view of the much greater intensity of solar radiation at $1200 \mathrm{~A}$. than at the shorter wave-lengths, that the mechanism here established is by far the predominant one.

The production of nitrogen atoms by predissociation in the $a-X$ bands is independent of the correctness of the value of $D\left(\mathrm{~N}_{2}\right)$ assumed above. If one assumes Gaydon's value ${ }^{5}$, the predissociation would lead to two normal atoms rather than one normal and one excited atom. However, it may be mentioned that the type of predissociation observed points strongly to the lower dissociation energy. Experiments are in preparation to settle this question definitely.

\section{G. Herzberg}

Yerkes Observatory,

Williams Bay, Wisconsin.

$$
\text { Oct. } 29 .
$$

1 See Wu, T. Y., Phys. Rev., 66, 65 (1944).

${ }^{2}$ Herman, R., C.R. Acad. Sci. Paris, 217, 141 (1943); Ann. de Phys., (11), 20, 241 (1945).

Birge, R. T., and Hopfleld, J. J., Astrophys. J., 68, 257 (1928),

- Herzberg, G., Phys. Rev., 69, 362 (1946).

"Gaydon, A. G., "Dissociation Energies and Spectra of Diatomic Molecules" (London, 1947).

\section{Calculation of the Piezo-electric Constants of $\alpha$ - and $\beta$-Quartz}

$\alpha$-Quartz possesses only two piezo-electric constants, $\varepsilon_{11}$ and $\varepsilon_{14}$, given by the equations

$$
\left.\begin{array}{l}
p_{x}=\varepsilon_{11}\left(u_{x x}-u_{y y}\right)+\varepsilon_{14} u_{y z} \\
p_{y}=-\varepsilon_{11} u_{x y}-\varepsilon_{14} u_{x z} \\
p_{z}=0
\end{array}\right\},
$$

where $p_{x}, p_{y}, p_{z}$ are the electric moments per unit volume developed on strain along the axes of the crystal, and $u_{x x}, u_{x y}$, etc., the components of strain. $\beta$-Quartz, which possesses a higher symmetry, has only one constant, $\varepsilon_{14}$, the other, $\varepsilon_{11}$, being zero. A method of deriving the above equations from the structure of the crystal, that is, the co-ordinates of the atoms in the unit cell, is not known. While the earlier work of Curie (1882), Riecke ${ }^{2}$ (1892) and Kelvin ${ }^{3}$ (1893) is purely speculative, Gibbs ${ }^{4}$ (1926) calculated the piezo-electric modulus $\delta_{11}$ on the basis of his structure and obtained a value which is nearly five times too high. Moreover, he did not calculate the other constant, $\delta_{14}$, and did not show how the 